\title{
Turning phases into frequencies
}

\section{The concept of geometrical phase, hitherto largely an academic issue, seems unexpectedly to have led to a novel means of fine-tuning the frequency of a laser.}

IT cannot be often that brooding about the basis of quantum mechanics leads people to the invention of a practical device, but this is what appears to have happened to R. Simon (from the Institute of Mathematical Sciences at Madras) and H. J. Kimble and E.C.G. Sudarshan from the University of Texas at Austin. They have been seeking a more general understanding of 'Berry's phase', or geometrical phase as M. V. Berry from the University of Bristol would modestly prefer it to be known, and have hit on a scheme for finetuning the frequency of a laser (Phys. Rev. Lett. 61, 19; 1988).

The confusions surrounding this issue have very little to do with quantum mechanics, but are instead bound up with the shadowy character of the concept of phase. This is simply a consequence of the fact that phase as such is hardly ever a measured quantity, a number. It is true, of course, that every interference experiment is an implicit measurement of phase. The bands of illumination on a screen behind a pair of Young's slits illuminated with monochromatic light are nothing but measures of the relative phase of the radiation arriving by two different routes.

If the result were not so visually spectacular, people might find themselves actually measuring phases and subtracting them from one another. But, in the event, phase usually makes its appearance as an apparently adjustable parameter in, for example, the equation of a wave; secondary-school students can write down $\cos (\omega t+\phi)$ as readily as anybody, but $\phi$ is rarely endowed with physical significance.

That state of affairs can be readily changed. Berry himself (see Nature 326, 277; 1987) has given a neat account of the transport of polarized light along a coiled optical fibre, showing that the plane of polarization of a propagating light wave is rotated by an amount determined by the solid angle contained within the coneshaped object, generated by anchoring to the same origin all the unit vectors representing the direction of the optical fibre. To put it another way, circularly polarized light waves are shifted in phase, but the phase shifts are in opposite directions for right- and left-handed polarization. All this, says Berry, is strictly classical.

On the face of things, phase arises in quantum mechanics in exactly the same way as in elementary descriptions of waves, but there is a complication because of the way in which a wave function can be multiplied by any number of unit modulus (the number will in general be complex) without affecting its physical significance (because all that matters is the product of the wave function and its complex conjugate, which is the probability of finding a particle at some point in space).

This leads to two important notions, first that of gauge invariance as in quantum electrodynamics and, second, the Aharanov-Bohm effect by which the state of a wave function is affected by electromagnetic potentials that do not generate electric or magnetic forces on the system as such. The neatest illustration is that of a Young's slit interference system for electrons with a long thin solenoid carrying a current placed symmetrically between the slits; the magnetic forces can be made indefinitely small by making the solenoid longer, but the electromagnetic potential (normally unobservable) remains unchanged, so that the interference pattern is distorted.

Berry's version of the geometrical phase is not so much a generalization as an independent but similar observation of what will happen to a quantum system if some parameter involved in its definition is slowly changed, which may occur for a host of reasons. The varying parameter may, for example, represent the strength of some externally imposed field or even the strength of the interaction between one part of the system and the rest. If the varying parameter eventually returns to its original value, so too will the quantum system return to its original state, but the phase will in general be different, and again be determined by a solid angle measuring the expanse of the track of the changing parameter in some kind of parameter space. Presumably what this means is that the state of a system, although not its actual physical properties, is marked by its recent history.

During the past year, several people have sought further generalizations of this notion. One of these is the generalization due to Aharanov and Anandan which, as Simon and his colleagues put it, "liberates" the geometric phase from the requirement that changes of the controlling parameter should be slow by the standards of the timescales characteristic of the system itself. Their trick was simply to recognize that the state of a quantum system can be represented as a vector in the infinite space of an acceptable set of eigenfunctions, and that the vector must trace out some closed circuit whose characteristics can be related to the externally imposed changes, whether quick or slow.

How does this relate to the fine-tuning of the frequency of a laser? This is how the argument goes. First, you construct a kind of Michelson's interferometer with input from a helium-neon laser. Use a beamsplitter to direct the laser beam into two orthogonal tracks, with a mirror at each end, and use one of the arms as a means of measuring the phase of the light beam in the other arm. Then arrange that the input source of light is plane polarized in a known direction, most simply by interposing a half-wave plate. Then the fun begins.

The business arm of the interferometer must be fitted with two quarter-wave plates, the first of which is set with its slow axis at an angle of $45^{\circ}$ to the plane of polarization. The output from that device is circularly polarized light, which is then channelled through a second quarterwave plate rotating slowly and at a constant rate on its axis. Because of the mirror at the end of the arm, the light beam of course makes two circuits of the course before interfering (or not, as the case may be) with the reference beam.

Simon and his colleagues note that this is essentially a system with quantum states - the states of polarization - which is influenced by an externally varied parameter, the position angle of the second quarter-wave plate which controls the state of polarization of the light beam in the several sections of the business arms of the interferometer. The argument quickly leads to the conclusion that the frequency of the light output from the interferometer is shifted by an amount determined by the rate of rotation of the quarter-wave plate.

That there should be a connection between phase and frequency is not surprising; both, after all, turn up together in any formula that describes a wave. But on this occasion, the usual phenomenon of beating does not occur. The real interest of the exercise, as conducted by Simon and his colleagues, is their use of a neat way of representing states of polarization as points on the surface of a sphere (originally devised by Poincaré, as it happens) which is also a neat way of getting to grips with the SU(2) group, famed for its role in the electro-weak interaction.

John Maddox 